\title{
Linear Independent Solutions and Operational Representations for Hypergeometric Functions of Four Variables
}

\author{
Maged G. Bin-Saad ${ }^{1}$ and Anvar Hasanov ${ }^{2}$ \\ ${ }^{1}$ Department of Mathematics, Aden University, P.O. Box 6014, Khormaksar, Yemen \\ ${ }^{2}$ Institute of Mathematics, 29 F. Khodjaev Street, 700125 Tashkent, Uzbekistan \\ Correspondence should be addressed to Maged G. Bin-Saad; mgbinsaad@yahoo.com
}

Received 6 February 2014; Accepted 26 May 2014; Published 15 June 2014

Academic Editor: Xiao-wei Gao

Copyright (c) 2014 M. G. Bin-Saad and A. Hasanov. This is an open access article distributed under the Creative Commons Attribution License, which permits unrestricted use, distribution, and reproduction in any medium, provided the original work is properly cited.

\begin{abstract}
In investigation of boundary-value problems for certain partial differential equations arising in applied mathematics, we often need to study the solution of system of partial differential equations satisfied by hypergeometric functions and find explicit linearly independent solutions for the system. Here we choose the Exton function $K_{2}$ among his 21 functions to show how to find the linearly independent solutions of partial differential equations satisfied by this function $K_{2}$. Based upon the classical derivative and integral operators, we introduce a new operational images for hypergeometric function $K_{2}$. By means of these operational images, a number of finite series and decomposition formulas are then found.
\end{abstract}

\section{Introduction and Preliminaries}

Solutions of many applied problems involving thermal conductivity and dynamics, electromagnetic oscillation and aerodynamics, and quantum mechanics and potential theory are obtainable with the help of hypergeometric (higher and special or transcendent) functions [1-4]. Such kinds of functions are often referred to as special functions of mathematical physics. They mainly appear in the solution of partial differential equations which are dealt with harmonic analysis method (see [5]). In view of various applications, it is interesting in itself and seems to be very important to conduct a continuous research of multiple hypergeometric functions. For instance, in [6], a comprehensive list of hypergeometric functions of three variables as many as 205 is recorded, together with their regions of convergence. It is noted that Riemann functions and the fundamental solutions of the degenerate second-order partial differential equations are expressible by means of hypergeometric functions of several variables (see [7-19]). Therefore, in investigation of boundary-value problems for these partial differential equations, we need to study the solution of the system of hypergeometric functions and find explicit linearly independent solutions (see $[12-17,19])$. Exton [20, pages 78-79] introduced 21 complete hypergeometric functions $K_{1}, K_{2}, \ldots, K_{21}$ of four variables. In [21] Sharma and Parihar introduced 83 complete hypergeometric functions $F_{1}^{(4)}, F_{2}^{(4)}, \ldots, F_{83}^{(4)}$ of four variables. It is remarked that, out of these 83 functions, the following 19 functions had already appeared in the work of Exton [20] in the different notations:

$$
\begin{array}{r}
F_{9}^{(4)}=K_{1}, \quad F_{1}^{(4)}=K_{2}, \quad F_{38}^{(4)}=K_{3}, \\
F_{10}^{(4)}=K_{4}, \quad F_{2}^{(4)}=K_{5}, \quad F_{59}^{(4)}=K_{6}, \\
F_{39}^{(4)}=K_{7}, \quad F_{11}^{(4)}=K_{8}, \quad F_{12}^{(4)}=K_{9}, \\
F_{3}^{(4)}=K_{10}, \quad F_{60}^{(4)}=K_{11}, \quad F_{40}^{(4)}=K_{12}, \\
F_{13}^{(4)}=K_{13}, \quad F_{77}^{(4)}=K_{14}, \quad F_{78}^{(4)}=K_{15}, \\
F_{79}^{(4)}=K_{16}, \quad F_{82}^{(4)}=K_{19}, \\
F_{81}^{(4)}=K_{20}, \quad F_{83}^{(4)}=K_{21} .
\end{array}
$$


Each quadruple hypergeometric function is of the form

$$
F^{(4)}(\cdot)=\sum_{m, n, p, q=0}^{\infty} \Delta(m, n, p, q) \frac{x^{m}}{m !} \frac{y^{n}}{n !} \frac{z^{p}}{p !} \frac{t^{q}}{q !},
$$

where $\Delta(m, n, p, q)$ is a certain sequence of complex parameters and there are twelve parameters in each function $F^{(4)}(\cdot)$. Here, for example, we choose the Exton function $K_{2}$ among his twenty-one functions

$$
\begin{aligned}
K_{2} & {\left[a, a, a, a, b, c, b, b ; e_{1}, e_{2}, e_{3}, e_{4} ; x, y, z, t\right] } \\
& =\sum_{m, n, p, q=0}^{\infty} \frac{(a)_{m+n+p+q}(b)_{m+n+q}(c)_{p}}{\left(e_{1}\right)_{m}\left(e_{2}\right)_{n}\left(e_{3}\right)_{p}\left(e_{4}\right)_{q}} \frac{x^{m}}{m !} \frac{y^{n}}{n !} \frac{z^{p}}{p !} \frac{t^{q}}{q !}
\end{aligned}
$$

to find the linearly independent solutions of partial differential equations satisfied by this function $K_{2}$.

\section{The System of Partial Differential Equations for $K_{2}$}

According to the theory of multiple hypergeometric functions (see [22]), the system of partial differential equations for Exton hypergeometric function $K_{2}$ is readily seen to be given as follows:

$$
\begin{aligned}
& \left(e_{1}+x \frac{\partial}{\partial x}\right)\left(x \frac{\partial}{\partial x}+1\right) x^{-1} u \\
& -\left(a+x \frac{\partial}{\partial x}+y \frac{\partial}{\partial y}+z \frac{\partial}{\partial z}+t \frac{\partial}{\partial t}\right) \\
& \times\left(b+x \frac{\partial}{\partial x}+y \frac{\partial}{\partial y}+t \frac{\partial}{\partial t}\right) u=0, \\
& \left(e_{2}+y \frac{\partial}{\partial y}\right)\left(y \frac{\partial}{\partial y}+1\right) y^{-1} u \\
& -\left(a+x \frac{\partial}{\partial x}+y \frac{\partial}{\partial y}+z \frac{\partial}{\partial z}+t \frac{\partial}{\partial t}\right) \\
& \times\left(b+x \frac{\partial}{\partial x}+y \frac{\partial}{\partial y}+t \frac{\partial}{\partial t}\right) u=0, \\
& \left(e_{3}+z \frac{\partial}{\partial z}\right)\left(z \frac{\partial}{\partial z}+1\right) z^{-1} u \\
& -\left(a+x \frac{\partial}{\partial x}+y \frac{\partial}{\partial y}+z \frac{\partial}{\partial z}+t \frac{\partial}{\partial t}\right)\left(c+z \frac{\partial}{\partial z}\right) u=0, \\
& \left(e_{4}+t \frac{\partial}{\partial t}\right)\left(t \frac{\partial}{\partial t}+1\right) t^{-1} u \\
& -\left(a+x \frac{\partial}{\partial x}+y \frac{\partial}{\partial y}+z \frac{\partial}{\partial z}+t \frac{\partial}{\partial t}\right) \\
& \times\left(b+x \frac{\partial}{\partial x}+y \frac{\partial}{\partial y}+t \frac{\partial}{\partial t}\right) u=0,
\end{aligned}
$$

where $u=K_{2}\left[a, b, c ; e_{1}, e_{2}, e_{3}, e_{4} ; x, y, z, t\right]$.
Now by making use of some elementary calculations, we find the following system of second-order partial differential equations:

$$
\begin{aligned}
& x(1-x) u_{x x}-y^{2} u_{y y}-t^{2} u_{t t}-2 x y u_{x y} \\
& -x z u_{x z}-2 x t u_{x t}-y z u_{y z}-2 y t u_{y t}-z t u_{z t} \\
& +\left[e_{1}-(a+b+1) x\right] u_{x}-(a+b+1) y u_{y} \\
& -b z u_{z}-(a+b+1) t u_{t}-a b u=0, \\
& y(1-y) u_{y y}-x^{2} u_{x x}-t^{2} u_{t t}-2 x y u_{x y}-x z u_{x z} \\
& -2 x t u_{x t}-y z u_{y z}-2 y t u_{y t}-z t u_{z t} \\
& -(a+b+1) x u_{x}+\left[e_{2}-(a+b+1) y\right] u_{y} \\
& -b z u_{z}-(a+b+1) t u_{t}-a b u=0, \\
& z(1-z) u_{z z}-x z u_{x z}-y z u_{y z}-z t u_{z t}-c x u_{x} \\
& -c y u_{y}+\left[e_{3}-(a+c+1) z\right] u_{z}-c t u_{t}-a c u=0 \text {, } \\
& t(1-t) u_{t t}-x^{2} u_{x x}-y^{2} u_{y y}-2 x y u_{x y}-x z u_{x z} \\
& -2 x t u_{x t}-y z u_{y z}-2 y t u_{y t}-z t u_{z t} \\
& -(a+b+1) x u_{x}-(a+b+1) y u_{y}-b z u_{z} \\
& +\left[e_{4}-(a+b+1) t\right] u_{t}-a b u=0 .
\end{aligned}
$$

It is noted that three equations of the system (5) are linearly dependent, because the hypergeometric function $K_{2}$ satisfies the system. Now, in order to find the linearly independent solutions of the system (5), we consider $u$ as in the form $u=x^{\alpha} y^{\beta} z^{\gamma} t^{\delta} w$ an unknown function, and $\alpha, \beta, \gamma$, and $\delta$ are constants which are to be determined. So, substituting $u=x^{\alpha} y^{\beta} z^{\gamma} t^{\delta} w$ into the system (5), we obtain

$$
\begin{aligned}
x(1- & x) w_{x x}-y^{2} w_{y y}-t^{2} w_{t t}-2 x y w_{x y}-x z w_{x z} \\
& -2 x t w_{x t}-y z w_{y z}-2 y t w_{y t}-z t w_{z t} \\
& +\left[E_{1}-(A+B+1) x\right] w_{x}-(A+B+1) y w_{y} \\
& -B z w_{z}-(A+B+1) t w_{t} \\
& +\left[\alpha\left(\alpha-1+e_{1}\right) x^{-1}-A B\right] w=0, \\
y(1- & y) w_{y y}-x^{2} w_{x x}-t^{2} w_{t t}-2 x y w_{x y} \\
& -x z w_{x z}-2 x t w_{x t}-y z w_{y z}-2 y t w_{y t}-z t w_{z t} \\
& -(A+B+1) x w_{x}+\left[E_{2}-(A+B+1) y\right] w_{y}
\end{aligned}
$$




$$
\begin{aligned}
& -B z w_{z}-(A+B+1) t w_{t} \\
+ & {\left[\beta\left(\beta-1+e_{2}\right) y^{-1}-A B\right] w=0, } \\
z(1- & z) w_{z z}-x z w_{x z}-y z w_{y z}-z t w_{z t}-C x w_{x} \\
& -C y w_{y}+\left[E_{3}-(A+C+1) z\right] w_{z}-C t w_{t} \\
+ & {\left[\gamma\left(\gamma-1+e_{3}\right) z^{-1}-A C\right] w=0, } \\
t(1- & t) w_{t t}-x^{2} w_{x x}-y^{2} w_{y y}-2 x y w_{x y}-x z w_{x z} \\
- & 2 x t w_{x t}-y z w_{y z}-2 y t w_{y t}-z t w_{z t} \\
- & (A+B+1) x w_{x}-(A+B+1) y w_{y} \\
- & B z w_{z}+\left[E_{4}-(A+B+1) t\right] w_{t} \\
+ & {\left[\delta\left(\delta-1+e_{4}\right) t^{-1}-A B\right] w=0, }
\end{aligned}
$$

where

$$
\begin{gathered}
A=\alpha+\beta+\gamma+\delta+a, \quad B=\alpha+\beta+\delta+b, \\
C=\gamma+c, \quad E_{1}=2 \alpha+e_{1}, \quad E_{2}=2 \beta+e_{2}, \\
E_{3}=2 \gamma+e_{3}, \quad E_{4}=2 \delta+e_{4} .
\end{gathered}
$$

\begin{tabular}{|c|c|c|c|c|c|c|c|c|}
\hline & 1 & 2 & 3 & 4 & 5 & 6 & 7 & 8 \\
\hline$o$ & \begin{tabular}{l|ll}
$:$ & 0 & 1
\end{tabular} & $1-e_{1}$ & 0 & 0 & 0 & $1-e_{1} 1$ & $1-e_{1} 1$ & $1-e_{1}$ \\
\hline F & 0 & 0 & $1-e_{2}$ & 0 & 0 & $1-e_{2}$ & 0 & 0 \\
\hline j & $: 0$ & 0 & 0 & $1-e_{3}$ & 0 & 0 & $1-e_{3}$ & 0 \\
\hline$\delta$ & 0 & 0 & 0 & 0 & $1-e_{4}$ & 0 & 0 & $1-e_{4}$ \\
\hline & 9 & 10 & 11 & 12 & 13 & 14 & 15 & 16 \\
\hline$\alpha:$ & 0 & 0 & 0 & $1-e_{1}$ & $11-e_{1}$ & $11-e_{1}$ & 0 & $1-e_{1}$ \\
\hline$\beta:$ & $1-e_{2}$ & $21-e_{2}$ & 0 & $1-e_{2}$ & $21-e_{2}$ & 0 & $1-e_{2}$ & $21-e_{2}$ \\
\hline$\gamma:$ & $1-e_{3}$ & 0 & $1-e_{3}$ & ${ }_{3} 1-e_{3}$ & 0 & $1-e_{3}$ & ${ }_{3} 1-e_{3}$ & $31-e_{3}$ \\
\hline$\delta:$ & 0 & $1-e_{4}$ & $41-e_{4}$ & 0 & $1-e_{4}$ & ${ }_{4} 1-e_{4}$ & $41-e_{4}$ & ${ }_{4} 1-e_{4}$ \\
\hline
\end{tabular}

It is noted that the system (6) is analogical to the system (5). Therefore, it is required that the following conditions should be satisfied:

$$
\begin{aligned}
& \alpha\left(\alpha-1+e_{1}\right)=0, \\
& \beta\left(\beta-1+e_{2}\right)=0, \\
& \gamma\left(\gamma-1+e_{3}\right)=0, \\
& \delta\left(\delta-1+e_{4}\right)=0 .
\end{aligned}
$$

(6)
It is not difficult to see that the system (8) satisfies the following solutions:
Finally, substituting all eight solutions (9) into (6), we find the following linearly independent solutions of the system (5):

$$
\begin{gathered}
u_{1}(x, y, z, t)=K_{2}\left(a, b, c ; e_{1}, e_{2}, e_{3}, e_{4} ; x, y, z, t\right), \\
u_{2}(x, y, z, t) \\
=x^{1-e_{1}} K_{2}\left[1-e_{1}+a, 1-e_{1}+b, c ;\right. \\
\left.2-e_{1}, e_{2}, e_{3}, e_{4} ; x, y, z, t\right], \\
u_{3}(x, y, z, t) \\
=y^{1-e_{2}} K_{2}\left[1-e_{2}+a, 1-e_{2}+b, c ;\right. \\
\left.e_{1}, 2-e_{2}, e_{3}, e_{4} ; x, y, z, t\right] \\
u_{4}(x, y, z, t) \\
=z^{1-e_{3}} K_{2}\left[1-e_{3}+a, b, 1-e_{3}+c ;\right. \\
\left.e_{1}, e_{2}, 2-e_{3}, e_{4} ; x, y, z, t\right]
\end{gathered}
$$

$$
\begin{aligned}
& u_{5}(x, y, z, t) \\
& =t^{1-e_{4}} K_{2}\left[1-e_{4}+a, 1-e_{4}+b, c ;\right. \\
& \left.e_{1}, e_{2}, e_{3}, 2-e_{4} ; x, y, z, t\right],
\end{aligned}
$$$$
u_{6}(x, y, z, t)
$$$$
=x^{1-e_{1}} y^{1-e_{2}}
$$$$
\times K_{2}\left[2-e_{1}-e_{2}+a, 2-e_{1}-e_{2}+b, c ;\right.
$$$$
\left.2-e_{1}, 2-e_{2}, e_{3}, e_{4} ; x, y, z, t\right],
$$$$
u_{7}(x, y, z, t)
$$$$
=x^{1-e_{1}} z^{1-e_{3}}
$$$$
\times K_{2}\left[2-e_{1}-e_{3}+a, 1-e_{1}+b, 1-e_{3}+c ;\right.
$$$$
\left.2-e_{1}, e_{2}, 2-e_{3}, e_{4} ; x, y, z, t\right],
$$ 


$$
\begin{aligned}
& u_{8}(x, y, z, t) \\
& =x^{1-e_{1}} t^{1-e_{4}} \\
& \times K_{2}\left[2-e_{1}-e_{4}+a, 2-e_{1}-e_{4}+b, c ;\right. \\
& \left.2-e_{1}, e_{2}, e_{3}, 2-e_{4} ; x, y, z, t\right] \text {, } \\
& u_{9}(x, y, z, t) \\
& =y^{1-e_{2}} z^{1-e_{3}} \\
& \times K_{2}\left[2-e_{2}-e_{3}+a, 1-e_{2}+b, 1-e_{3}+c ;\right. \\
& \left.e_{1}, 2-e_{2}, 2-e_{3}, e_{4} ; x, y, z, t\right] \text {, } \\
& u_{10}(x, y, z, t) \\
& =y^{1-e_{2}} t^{1-e_{4}} \\
& \times K_{2}\left[2-e_{2}-e_{4}+a, 2-e_{2}-e_{4}+b, c ;\right. \\
& \left.e_{1}, 2-e_{2}, e_{3}, 2-e_{4} ; x, y, z, t\right] \text {, } \\
& u_{11}(x, y, z, t) \\
& =z^{1-e_{3}} t^{1-e_{4}} \\
& \times K_{2}\left[2-e_{3}-e_{4}+a, 1-e_{4}+b, 1-e_{3}+c ;\right. \\
& \left.e_{1}, e_{2}, 2-e_{3}, 2-e_{4} ; x, y, z, t\right], \\
& u_{12}(x, y, z, t) \\
& =x^{1-e_{1}} y^{1-e_{2}} z^{1-e_{3}} \\
& \times K_{2}\left[3-e_{1}-e_{2}-e_{3}+a, 2-e_{1}-e_{2}+b, 1-e_{3}+c ;\right. \\
& \left.2-e_{1}, 2-e_{2}, 2-e_{3}, e_{4} ; x, y, z, t\right], \\
& u_{13}(x, y, z, t) \\
& =x^{1-e_{1}} y^{1-e_{2}} t^{1-e_{4}} \\
& \times K_{2}\left[3-e_{1}-e_{2}-e_{4}+a, 3-e_{1}-e_{2}-e_{4}+b, c ;\right. \\
& \left.2-e_{1}, 2-e_{2}, e_{3}, 2-e_{4} ; x, y, z, t\right], \\
& u_{14}(x, y, z, t) \\
& =x^{1-e_{1}} z^{1-e_{3}} t^{1-e_{4}} \\
& \times K_{2}\left[3-e_{1}-e_{3}-e_{4}+a, 2-e_{1}-e_{4}+b, 1-e_{3}+c ;\right. \\
& \left.2-e_{1}, e_{2}, 2-e_{3}, 2-e_{4} ; x, y, z, t\right], \\
& u_{15}(x, y, z, t) \\
& =y^{1-e_{2}} z^{1-e_{3}} t^{1-e_{4}} \\
& \times K_{2}\left[3-e_{2}-e_{3}-e_{4}+a, 2-e_{2}-e_{4}+b, 1-e_{3}+c ;\right. \\
& \left.e_{1}, 2-e_{2}, 2-e_{3}, 2-e_{4} ; x, y, z, t\right],
\end{aligned}
$$

$$
\begin{aligned}
& u_{16}(x, y, z, t) \\
& \begin{array}{l}
=x^{1-e_{1}} y^{1-e_{2}} z^{1-e_{3}} t^{1-e_{4}} \\
\quad \times K_{2}\left[4-e_{1}-e_{2}-e_{3}-e_{4}+a,\right. \\
\quad 3-e_{1}-e_{2}-e_{4}+b, 1-e_{3}+c ; \\
\left.\quad 2-e_{1}, 2-e_{2}, 2-e_{3}, 2-e_{4} ; x, y, z, t\right] .
\end{array}
\end{aligned}
$$

Moreover, it is seen that the global solution of the system (5) is combined to be in the form

$$
u=\sum_{j=1}^{16} k_{j} u_{j}
$$

where $k_{i}(i=1,2, \ldots, 16)$ are constants.

\section{Operational Representations for $K_{2}$ and Applications}

In this section, we apply the concept of the right RiemannLiouville fractional derivative to obtain operational images for hypergeometric functions $K_{2}$. Indeed, in this section we build up the right Riemann-Liouville fractional derivative operator, which plays the role of augmenting parameters in the hypergeometric functions involved (see [23, Chapters 4 and 5]). In particular, we will deal with operational definitions ruled by the operators $\widehat{D}_{x}$ and $\widehat{D}_{x}^{-1}$ where $\widehat{D}_{x}$ denotes the derivative operator and $\widehat{D}_{x}^{-1}$ defines the inverse of the derivative and once acting on unity, it yields

$$
\widehat{D}_{x}^{-m} 1=\frac{x^{m}}{m !}, \quad m \in N \cup\{0\} .
$$

It is evident that $D_{x}^{-1}$ is essentially an integral operator and the lower limit has been assumed to be zero. The following two formulas are well-known consequences of the derivative operator $\widehat{D}_{x}$ and the integral operator $\widehat{D}_{x}^{-1}$ (see [24]). Consider

$$
\begin{aligned}
& \widehat{D}_{x}^{m} x^{\lambda}=\frac{\Gamma(\lambda+1)}{\Gamma(\lambda-m+1)} x^{\lambda-m}, \\
& \widehat{D}_{x}^{-m} x^{\lambda}=\frac{\Gamma(\lambda+1)}{\Gamma(\lambda+m+1)} x^{\lambda+m},
\end{aligned}
$$

where $m \in N \cup\{0\}, \lambda \in C /\{-1,-2, \ldots\}$. Based on the operational relations (13), we have

$$
\widehat{D}_{t}^{m} \widehat{D}_{u}^{-m}\left\{t^{\beta+m-1} u^{\gamma-1}\right\}=\frac{(\beta)_{m}}{(\gamma)_{m}}\left\{t^{\beta-1} u^{\gamma+m-1}\right\} .
$$

In view of the definition of the Gaussian hypergeometric function ${ }_{2} F_{1}[6]$,

$$
{ }_{2} F_{1}[\alpha, \beta ; \gamma ; x]=\sum_{n=0}^{\infty} \frac{(\alpha)_{n}(\beta)_{n}}{(\gamma)_{n} n !} x^{n} .
$$

Bin-Saad [25] introduced the following operational representations for Gaussian hypergeometric ${ }_{2} F_{1}$. 
Lemma 1. Let $\operatorname{Re}(\beta)>0$ and $\operatorname{Re}(\gamma)>0$; then

$$
\begin{gathered}
\left(1-x \widehat{D}_{t} u^{-1} \widehat{D}_{u}^{-1} t\right)^{-\alpha}\left\{t^{\beta-1} u^{\gamma-1}\right\}=\left\{t^{\beta-1} u^{\gamma-1}\right\}_{2} F_{1}[\alpha, \beta ; \gamma ; x], \\
\left(1-\widehat{D}_{t} \widehat{D}_{x}^{-1} t\right)^{-\alpha}\left\{t^{\beta-1} x^{\gamma-1}\right\}=\left\{t^{\beta-1} x^{\gamma-1}\right\}_{2} F_{1}[\alpha, \beta ; \gamma ; x] .
\end{gathered}
$$

For the quadruple hypergeometric function $K_{2}$, one introduces the following operational representations.

Theorem 2. Let $\operatorname{Re}(a)>0, \operatorname{Re}(b)>0, \operatorname{Re}(c)>0, \operatorname{Re}\left(e_{1}\right)>0$, $\operatorname{Re}\left(e_{2}\right)>0, \operatorname{Re}\left(e_{3}\right)>0$, and $\operatorname{Re}\left(e_{4}\right)>0$; then

$$
\begin{aligned}
(1- & \left.x \widehat{D}_{t_{1}} t_{2}^{-1} \widehat{D}_{t_{2}}^{-1} t_{1}-y \widehat{D}_{t_{1}} t_{3}^{-1} \widehat{D}_{t_{3}}^{-1} t_{1}-u \widehat{D}_{t_{1}} t_{5}^{-1} \widehat{D}_{t_{5}}^{-1} t_{1}\right)^{-b} \\
& \times\left(1-z \widehat{D}_{t_{1}} t_{4}^{-1} \widehat{D}_{t_{4}}^{-1} t_{1}\right)^{-c}\left\{t_{1}^{a-1} t_{2}^{e_{1}-1} t_{3}^{e_{2}-1} t_{3}^{e_{3}-1} t_{5}^{e_{4}-1}\right\} \\
= & \left\{t_{1}^{a-1} t_{2}^{e_{1}-1} t_{3}^{e_{2}-1} t_{4}^{e_{3}-1} t_{5}^{e_{4}-1}\right\} \\
& \times K_{2}\left[a, a, a, a ; b, b, c, b ; e_{1}, e_{2}, e_{3}, e_{4} ; x, y, z, u\right], \\
(1- & x \widehat{D}_{t_{1}} t_{3}^{-1} \widehat{D}_{t_{3}}^{-1} t_{1}-y \widehat{D}_{t_{1}} t_{4}^{-1} \widehat{D}_{t_{4}}^{-1} t_{1} \\
- & \left.z \widehat{D}_{t_{2}} t_{5}^{-1} \widehat{D}_{t_{5}}^{-1} t_{2}-u \widehat{D}_{t_{1}} t_{6}^{-1} \widehat{D}_{t_{6}}^{-1} t_{1}\right)^{-a} \\
& \times\left\{t_{1}^{b-1} t_{2}^{c-1} t_{3}^{e_{1}-1} t_{4}^{e_{2}-1} t_{5}^{e_{3}-1} t_{6}^{e_{4}-1}\right\} \\
= & \left\{t_{1}^{b-1} t_{2}^{c-1} t_{3}^{e_{1}-1} t_{4}^{e_{2}-1} t_{5}^{e_{3}-1} t_{6}^{e_{4}-1}\right\} \\
& \times K_{2}\left[a, a, a, a ; b, b, c, b ; e_{1}, e_{2}, e_{3}, e_{4} ; x, y, z, u\right] .
\end{aligned}
$$

Proof. Denote, for convenience, the left-hand side of assertion (17) by $I$. Then, as a consequence of the binomial theorem, it is easily seen that

$$
\begin{aligned}
I= & \sum_{m, n, p, q=0}^{\infty} \frac{(b)_{m+n+q}(c)_{p} x^{m} y^{n} z^{p} u^{q} t_{2}^{-m} t_{3}^{-n} t_{4}^{-p} t_{5}^{-q}}{m ! n ! p ! q !} \\
& \times \widehat{D}_{t_{1}}^{m+n+p+q} \widehat{D}_{t_{2}}^{-m} \widehat{D}_{t_{3}}^{-n} \widehat{D}_{t_{4}}^{-p} \widehat{D}_{t_{5}}^{-q} \\
& \times\left\{t_{1}^{a+m+n+p+q-1} t_{2}^{e_{1}-1} t_{3}^{e_{2}-1} t_{4}^{e_{3}-1} t_{5}^{e_{4}-1}\right\} .
\end{aligned}
$$

Upon using (14) and considering definition (3), we are led finally to right-hand side of assertion (17). The proof of assertion (18) runs parallel to that of assertion (17); then we skip the details.

One of the advantages offered by the use of the operational images (17) and (18) is the possibility of establishing finite sums for the quadruple hypergeometric series $K_{2}$. First, let, for convenience,

$$
\begin{aligned}
\widehat{M}= & x \widehat{D}_{t_{1}} t_{3}^{-1} \widehat{D}_{t_{3}}^{-1} t_{1}-y \widehat{D}_{t_{1}} t_{4}^{-1} \widehat{D}_{t_{4}}^{-1} t_{1} \\
& -z \widehat{D}_{t_{2}} t_{5}^{-1} \widehat{D}_{t_{5}}^{-1} t_{2}-u \widehat{D}_{t_{1}} t_{6}^{-1} \widehat{D}_{t_{6}}^{-1} t_{1}, \\
\widehat{N}= & x \widehat{D}_{t_{1}} t_{2}^{-1} \widehat{D}_{t_{2}}^{-1} t_{1}-y \widehat{D}_{t_{1}} t_{3}^{-1} \widehat{D}_{t_{3}}^{-1} t_{1}-u \widehat{D}_{t_{1}} t_{5}^{-1} \widehat{D}_{t_{5}}^{-1} t_{1} \\
\widehat{R}= & z \widehat{D}_{t_{1}} t_{4}^{-1} \widehat{D}_{t_{4}}^{-1} t_{1} .
\end{aligned}
$$

By employing (18) and taking advantage from the identity

$$
\begin{gathered}
{[1-(1-\widehat{M})]^{n}\left\{t_{1}^{b-1} t_{2}^{c-1} t_{3}^{e_{1}-1} t_{4}^{e_{2}-1} t_{5}^{e_{3}-1} t_{6}^{e_{4}-1}\right\}} \\
=[\widehat{M}]^{n}\left\{t_{1}^{b-1} t_{2}^{c-1} t_{3}^{e_{1}-1} t_{4}^{e_{2}-1} t_{5}^{e_{3}-1} t_{6}^{e_{4}-1}\right\}
\end{gathered}
$$

and the definition of the general triple hypergeometric series $F^{(3)}[x, y, z]$ (see $[6]$ ), we can state that

$$
\begin{gathered}
\sum_{r=0}^{n}(-1)^{r}\left(\begin{array}{l}
n \\
r
\end{array}\right) K_{2}\left[-r,-r,-r,-r ; b, b, c, b ; e_{1}, e_{2}, e_{3}, e_{4} ; x, y, z, u\right] \\
\quad=\frac{(b)_{n}}{\left(e_{1}\right)_{n}} F^{(3)}\left[\begin{array}{l}
-n, 1-e_{1}-n::--; c ;--; \\
-------: e_{2} ; e_{3} ; e_{4} ;
\end{array} \frac{y}{x}, \frac{z}{x}, \frac{u}{x}\right] .
\end{gathered}
$$

Similarly, in view of (17), the definition of Appell hypergeometric series $F_{4}[x, y]$ (see $[6$, page 23 , equation (5)]) and the use of the following identity

$$
\begin{aligned}
& {[1-(1-\widehat{N})]^{n}[1-(1-\widehat{R})]^{m}\left\{t_{1}^{a-1} t_{2}^{e_{1}-1} t_{3}^{e_{2}-1} t_{4}^{e_{3}-1} t_{5}^{e_{4}-1}\right\}} \\
& =[\widehat{N}]^{n}[\widehat{R}]^{m}\left\{t_{1}^{a-1} t_{2}^{e_{1}-1} t_{3}^{e_{2}-1} t_{4}^{e_{3}-1} t_{5}^{e_{4}-1}\right\}
\end{aligned}
$$

allow to conclude that

$$
\begin{aligned}
\sum_{r=0}^{n} \sum_{s=0}^{m}(-1)^{r+s}\left(\begin{array}{l}
n \\
r
\end{array}\right)\left(\begin{array}{c}
m \\
s
\end{array}\right) \\
\quad \times K_{2}\left[a, a, a, a,-r,-r,-s,-r ; e_{1}, e_{2}, e_{3}, e_{4} ; x, y, z, u\right] \\
=\frac{(a)_{m+n}}{\left(e_{1}\right)_{n}\left(e_{3}\right)_{m}} F_{4}\left[-n, 1-e_{1}-n ; e_{2}, e_{4} ; \frac{y}{x}, \frac{u}{x}\right] .
\end{aligned}
$$

Also, by virtue of the identity

$$
\begin{aligned}
& {[(1-\widehat{N})]^{-b}[(1-\widehat{R})]^{-b}\left\{t_{1}^{a-1} t_{2}^{e_{1}-1} t_{3}^{e_{2}-1} t_{4}^{e_{3}-1} t_{5}^{e_{4}-1}\right\}} \\
& \quad=[1-\widehat{N}-\widehat{R}+\widehat{N} \widehat{R}]^{-b}\left\{t_{1}^{a-1} t_{2}^{e_{1}-1} t_{3}^{e_{2}-1} t_{4}^{e_{3}-1} t_{5}^{e_{4}-1}\right\},
\end{aligned}
$$

we find the following decomposition formula:

$$
\begin{aligned}
& \sum_{s, k, r=0}^{\infty} \frac{(a)_{2 s+2 k+2 r}(b)_{s+k+r}}{\left(e_{1}\right)_{s}\left(e_{2}\right)_{k}\left(e_{3}\right)_{s+k+r}\left(e_{4}\right)_{r}}(-x z)^{s}(-y z)^{k}(-u z)^{r} \\
& \times F_{C}^{(4)}[a+2 s+2 k+2 r, b+s+k+r ; \\
& \left.\quad e_{1}, e_{2}, e_{3}, e_{4} ; x, y, z, u\right] \\
& =K_{2}\left[a, a, a, a ; b, b, b, b ; e_{1}, e_{2}, e_{3}, e_{4} ; x, y, z, u\right],
\end{aligned}
$$

where $F_{C}^{(4)}$ is Lauricella function of four variables [6, page 33, equation (3)].

Moreover, in view of the composition rule

$$
\begin{aligned}
{[(1-\widehat{M})]^{-2 a} } & =[(1-\widehat{M})]^{-a}[(1-\widehat{M})]^{-a} \\
& =\left[1-2 \widehat{M}+\widehat{M}^{2}\right]^{-a}
\end{aligned}
$$


and the operational image (18), we easily find for the function $K_{2}$ that

$$
\begin{aligned}
& K_{2}\left[2 a, 2 a, 2 a, 2 a, b, b, c, b ; e_{1}, e_{2}, e_{3}, e_{4} ; x, y, z, u\right] \\
& =\sum_{m, n, p, q, s, r, k, l, h, t=0}^{\infty} \frac{(a)_{M}(b)_{N}(c)_{P}}{\left(e_{1}\right)_{Q^{(1)}}\left(e_{2}\right)_{Q^{(2)}}\left(e_{3}\right)_{Q^{(3)}}\left(e_{4}\right)_{Q^{(4)}}} \\
& \quad \times x^{2 m} y^{2 n} z^{2 p} u^{2 q}(2 x y)^{s}(2 x z)^{k}(2 x u)^{r} \\
& \quad \times(2 y z)^{l}(2 y u)^{h}(2 z u)^{t} \\
& \quad \times K_{2}[a+M, a+M, a+M, a+M, b+N, b+N, \\
& \quad c+N, b+N ; e_{1}+Q^{(1)}, \\
& \left.\quad e_{2}+Q^{(2)}, e_{3}+Q^{(3)}, e_{4}+Q^{(4)} ; x, y, z, u\right],
\end{aligned}
$$

where

$$
\begin{gathered}
M:=m+n+p+q+s+k+r+l+h+t, \\
P:=2 p+k+l+t, \\
N:=2(m+n+q+s+r+h)+k+l+t, \\
Q^{(1)}:=2 m+s+k+r, \\
Q^{(2)}:=2 n+s+l+h, \quad Q^{(3)}:=2 p+k+l+t, \\
Q^{(4)}:=2 q+r+h+t .
\end{gathered}
$$

Finally, let us stress that the schema suggested in Sections 2 and 3 can be applied to find linear independent solutions, symbolic operational images, finite sums, and decomposition formulas for other quadruple hypergeometric functions.

\section{Conflict of Interests}

The authors declare that they have no competing interests.

\section{References}

[1] L. Bers, Mathematical Aspects of Subsonic and Transonic Gas Dynamics, John Wiley \& Sons, New York, NY, USA, 1958.

[2] F. I. Frankl, Selected Works in Gas Dynamics, Nauka, Moscow, Russia, 1973.

[3] A. W. Niukkanen, "Generalised hypergeometric series ${ }^{\mathrm{N}} \mathrm{F}\left(\mathrm{x}_{1}, \ldots, \mathrm{x}_{\mathrm{N}}\right)$ arising in physical and quantum chemical applications," Journal of Physics A: Mathematical and General, vol. 16, no. 9, pp. 1813-1825, 1983.

[4] G. Lohofer, "Theory of an electromagnetically levitated metal sphere I: absorbed power," SIAM Journal on Applied Mathematics, vol. 49, no. 2, pp. 567-581, 1989.

[5] A. Erdélyi, W. Magnus, F. Oberhettinger, and F. G. Tricomi, Higher Transcendental Functions, vol. 1, McGraw-Hill, New York, NY, USA, 1953.

[6] H. M. Srivastava and P. W. Karlsson, Multiple Gaussian Hypergeometric Series, Halsted Press, Ellis Horwood, Chichester, UK; John Wiley \& Sons, New York, NY, USA, 1985.
[7] A. Altin, "Some expansion formulas for a class of singular partial differential equations," Proceedings of the American Mathematical Society, vol. 85, no. 1, pp. 42-46, 1982.

[8] J. Barros-Neto and I. M. Gelfand, "Fundamental solutions for the Tricomi operator," Duke Mathematical Journal, vol. 98, no. 3, pp. 465-483, 1999.

[9] J. Barros-Neto and I. M. Gelfand, "Fundamental solutions for the Tricomi operator II," Duke Mathematical Journal, vol. 111, no. 3, pp. 561-584, 2002.

[10] J. Barros-Neto and I. M. Gelfand, "Fundamental solutions of the Tricomi operator III," Duke Mathematical Journal, vol. 128, no. 1, pp. 119-140, 2005.

[11] A. J. Fryant, "Growth and complete sequences of generalized biaxially symmetric potentials," Journal of Differential Equations, vol. 31, no. 2, pp. 155-164, 1979.

[12] A. Hasanov, "Fundamental solutions of generalized bi-axially symmetric Helmholtz equation," Complex Variables and Elliptic Equations, vol. 52, no. 8, pp. 673-683, 2007.

[13] A. Hasanov, "Some solutions of generalized Rassias's equation," International Journal of Applied Mathematics and Statistics, vol. 8, no. 7, pp. 20-30, 2007.

[14] A. Hasanov, "The solution of the Cauchy problem for generalized Euler-Poisson-Darboux equation," International Journal of Applied Mathematics and Statistics, vol. 8, no. 7, pp. 30-44, 2007.

[15] A. Hasanov, "Fundamental solutions for degenerated elliptic equation with two perpendicular lines of degeneration," International Journal of Applied Mathematics and Statistics, vol. 13, no. 8, pp. 41-49, 2008.

[16] A. Hasanov, J. M. Rassias, and M. Turaev, "Fundamental solution for the generalized Elliptic Gellerstedt equation," in Functional Equations, Difference Inequalities and ULAM Stability Notions, vol. 6, pp. 73-83, Nova Science Publishers, New York, NY, USA, 2010.

[17] A. Hasanov and E. T. Karimov, "Fundamental solutions for a class of three-dimensional elliptic equations with singular coefficients," Applied Mathematics Letters, vol. 22, no. 12, pp. 1828-1832, 2009.

[18] P. A. McCoy, "Polynomial approximation and growth of generalized axisymmetric potentials," Canadian Journal of Mathematics, vol. 31, no. 1, pp. 49-59, 1979.

[19] M. S. Salakhitdinov and A. Hasanov, "A solution of the Neumann-Dirichlet boundary value problem for generalized bi-axially symmetric Helmholtz equation," Complex Variables and Elliptic Equations, vol. 53, no. 4, pp. 355-364, 2008.

[20] H. Exton, Multiple Hypergeometric Functions and Applications, Halsted Press, London, UK, 1976.

[21] C. Sharma and C. L. Parihar, "Hypergeometric functions of four variables," Indian Academy of Mathematics, vol. 11, pp. 121-133, 1989.

[22] P. Appell and J. Kampé de Fériet, Fonctions Hypergéométriques et Hypersphériques: Polynômes d'Hermite, Gauthier-Villars, Paris, France, 1926.

[23] H. M. Srivastava and H. L. Manocha, A Treatise on Generating Functions, Halsted Press, London, UK, 1984.

[24] K. S. Miller and B. Ross, An Introduction to the Fractional Calculus and Fractional Differential Equations, Wiley-Interscience, New York, NY, USA, 1993.

[25] M. G. Bin-Saad, "Symbolic operational images and decomposition formulas for hypergeometric functions," Journal of Mathematical Analysis and Applications, vol. 376, no. 2, pp. 451468, 2011. 


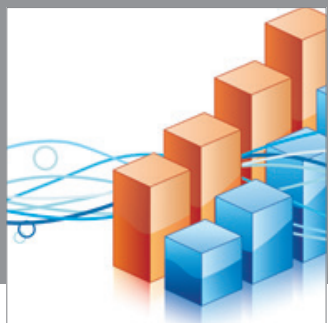

Advances in

Operations Research

mansans

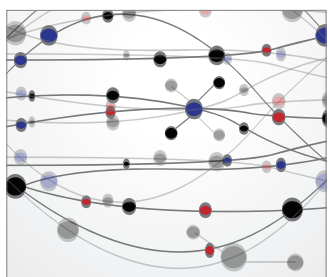

The Scientific World Journal
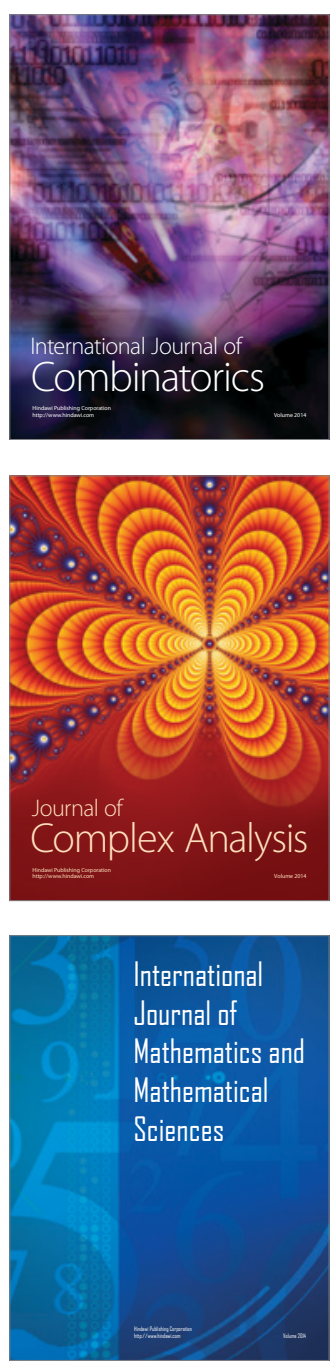
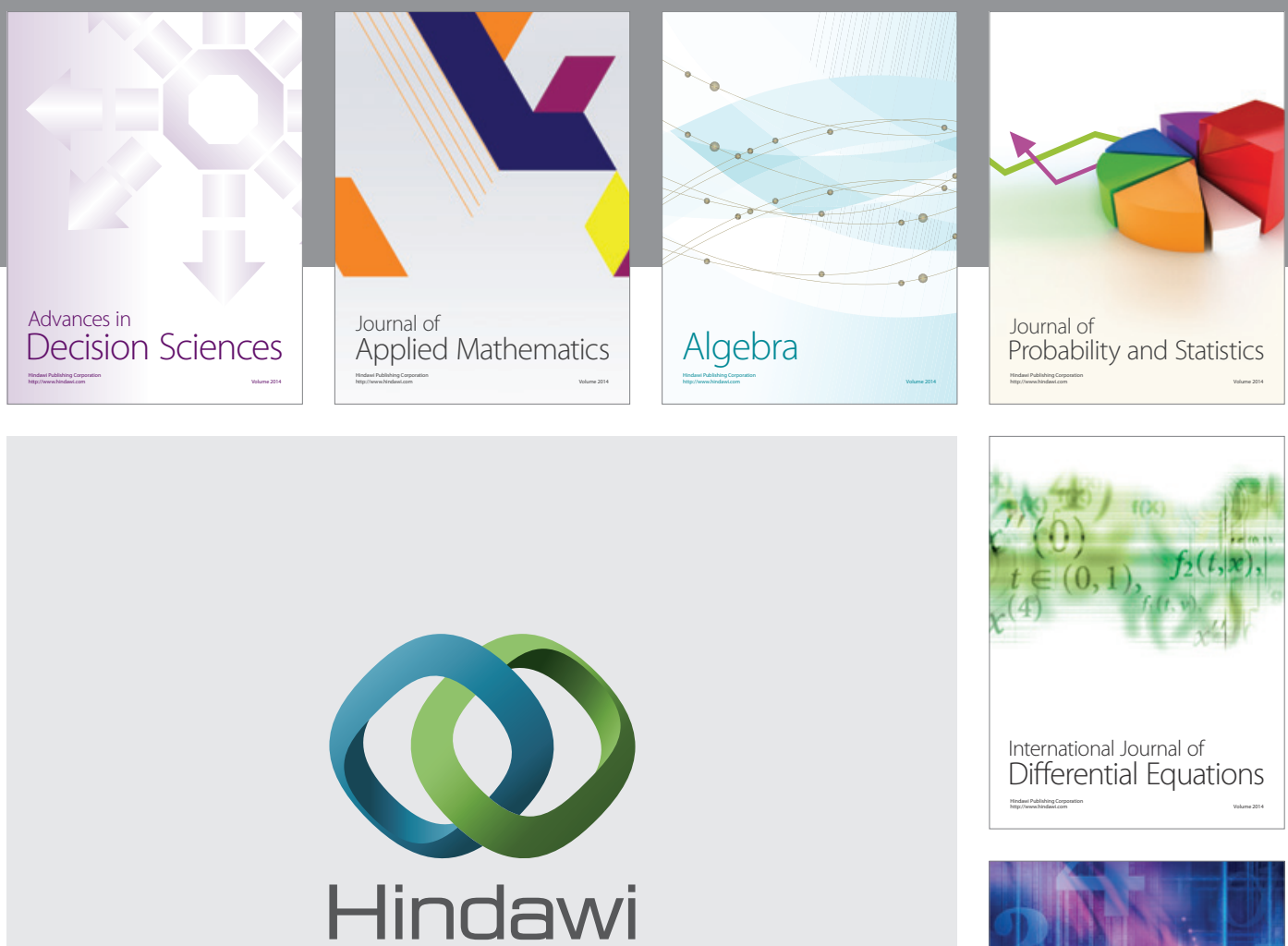

Submit your manuscripts at http://www.hindawi.com
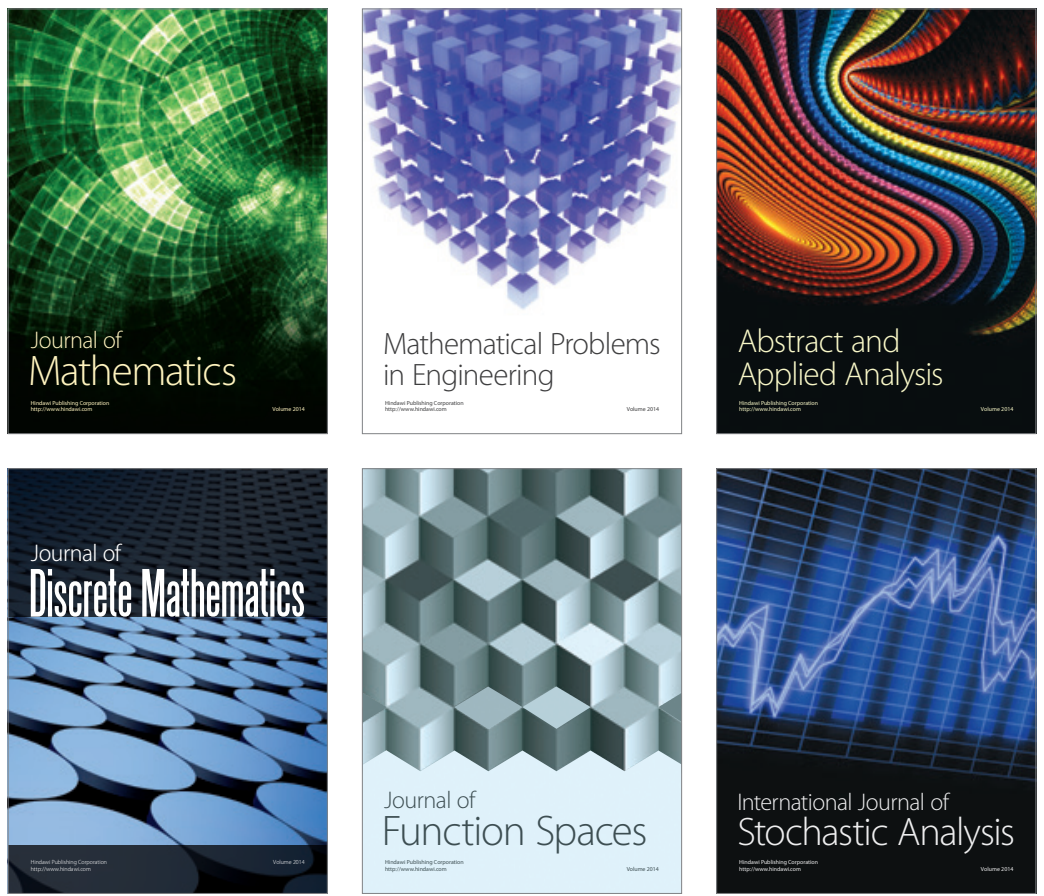

Journal of

Function Spaces

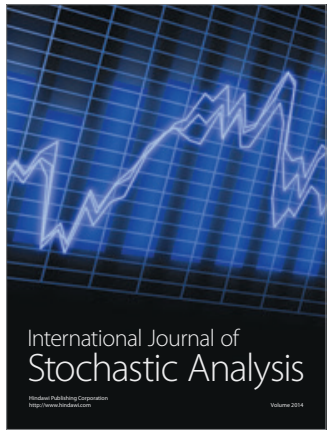

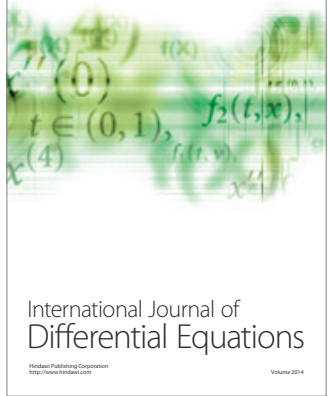
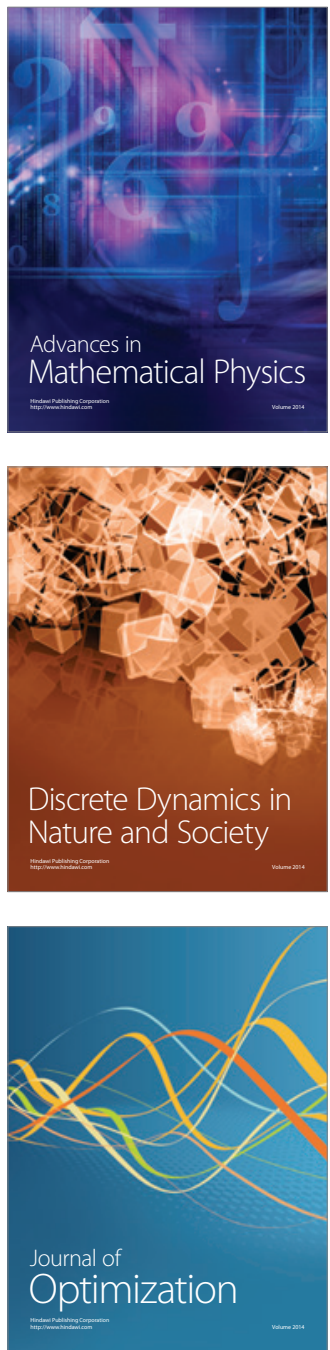